\title{
Generation of Antagonistic RNA Aptamers Specific to Proinflammatory Cytokine Interleukin-32
}

\author{
Seho Kim, ${ }^{\dagger}$ Jung-Hee Kim, ${ }^{\dagger}$ Sujin Yoon, ${ }^{\dagger}$ Keun-Sik Kim, ${ }^{\dagger \dagger}$ Moon-Young Yoon, ${ }^{\star}$ Do-Young Yoon, ${ }^{\dagger,}$ and Dong-Eun Kim ${ }^{\dagger,+*}$ \\ ${ }^{\dagger}$ Department of Bioscience and Biotechnology, ${ }^{\star}$ WCU Program, and ${ }^{\S}$ BMIC, Konkuk University, Seoul 143-701, Korea \\ ${ }^{*}$ Department of Chemistry, Hanyang University, Seoul 133-791, Korea \\ *E-mail: kimde@konkuk.ac.kr \\ Received September 9, 2010, Accepted September 24, 2010
}

\begin{abstract}
Interleukin 32 (IL-32) is a recently identified cytokine that induces major proinflammatory cytokines such as TNF $\alpha$ and IL-1 $\beta$, which play an important role in chronic inflammatory diseases. To antagonize the biological function of IL-32 in cells, we generated RNA aptamers that could bind specifically to IL-32 protein. The highest affinity aptamer, AC3-3, successfully antagonized IL-32 by abolishing the induction of TNF $\alpha$ in the human lung carcinoma cells expressing IL-32. This aptamer could be used as a potent and selective antagonist against IL-32 to further elucidate the roles of IL-32 in chronic inflammatory diseases, as well as a therapeutic agent.
\end{abstract}

Key Words: Interleukin-32, Proinflammatory cytokine, RNA aptamer, TNF $\alpha$

\section{Introduction}

Interleukin-32 (IL-32) is a newly identified proinflammatory cytokine that was originally recognized as a transcript expressed in activated natural killer cells stimulated with IL-2 or T cells activated with mitogens. ${ }^{1,2}$ IL-32 is expressed in blood monocytes, endothelial cells, and epithelial cells. ${ }^{2,3}$ Initially, IL-32 was identified as a reciprocal proinflammatory cytokine regulated by the proinflammatory cytokine, IL-18, which is associated with various chronic inflammatory diseases such as rheumatoid arthritis, psoriasis, and inflammatory bowel disease. ${ }^{4,5}$ IL-32 has emerged as an important factor involved in innate and adaptive immune responses because it stimulates monocytes to produce proinflammatory cytokines such as IL- $1 \beta$, IL- 6 , TNF $\alpha$, and chemokines $^{6}$ and induces differentiation of monocytes. ${ }^{7}$ The fulllength IL-32 gene of 705 bp has several splice variants including IL-32 $\alpha, \beta, \gamma, \delta, \varepsilon$ and $\zeta$, among which IL-32 $\alpha$ exists as the major isoform in hematopoietic cells. ${ }^{2,8}$ To date, receptors of those IL-32 and its variants have not been identified, except that proteinase 3 has recently been identified as an IL-32 binding protein. ${ }^{9}$

Recent studies have shown that IL-32 is highly expressed in rheumatoid arthritis, ${ }^{10,11}$ ulcerative colitis, ${ }^{6}$ human stomach cancer and lung cancer, ${ }^{12}$ indicating that cancer progression is promoted by inflammation. ${ }^{13}$ Overexpression of human IL-32 in mice by bone marrow transplantation (BM-hIL-32) was found to elicit the expression and secretion of TNF $\alpha$, IL-1 $\beta$, and IL-6 in spleen cells after lipopolysaccharide stimulation. ${ }^{10}$ In addition, IL-32 associated with elevated TNF $\alpha$ expression was found to exacerbate inflammatory arthritis and colitis. ${ }^{10}$ It has also been shown that a potent inducer of inflammation, prostaglandin E2, is induced by IL-32 in mouse macrophages and human blood monocytes. ${ }^{11}$ Knockdown of IL-32 suppresses expression of anti-apoptotic proteins such as Bcl-2 in pancreatic cancer cells ${ }^{14}$ and impairs the induction of TNF $\alpha$ and IL-1 $\beta$ in human monocytic cells. ${ }^{15}$ Importantly, inhibitory blockade of IL- $1 \beta$ and TNF $\alpha$ has been administered clinically to lessen the severity of chronic inflammatory diseases. ${ }^{16,17}$ Since IL-32 reciprocally induces various proinflammatory cytokines including IL-1 $\beta$, IL-18, TNF $\alpha$, and IL-6, blocking IL-32 with effective binding ligands could be beneficial to patients with chronic inflammatory diseases associated with the up-regulation of inflammatory cytokines. $^{15}$

Nucleic acids ligands that are produced using a procedure known as systematic evolution of ligands by exponential enrichment (SELEX) as an in vitro selection strategy can adopt complex structures (aptamers) to bind to target proteins with high affinities. ${ }^{18,19}$ The SELEX procedure is an efficient method for isolation of high affinity single stranded oligonucleotides from DNA and RNA libraries consisting of $10^{14} \sim 10^{15}$ random oligonucleotide sequences. In addition to protein targets, oligonucleotide ligands for target molecules with high affinities have been identified using the SELEX process for organic dyes and other small molecules. ${ }^{20}$ Similar to antibodies, nucleic acid aptamers fit epitopes on the target protein with nanomolar or subnanomolar affinities by distinguish them from other proteins. RNA or DNA aptamers against target proteins have been shown to have inhibitory effects. RNA aptamers generated against a member of the IL- 6 cytokine family have been identified as antagonistic ligands that interfere with binding of the cytokine to its cognate receptor in vitro. ${ }^{21}$

In the present study, we identified RNA aptamers that could specifically bind to the proinflammatory cytokine IL-32 to explore the function of endogenous IL-32 without influencing the expression of IL-32 protein in cells. Antagonistic RNA aptamers against IL-32 that were introduced into the cells reduced the expression of TNF $\alpha$ induced by IL-32. Thus, the antagonistic RNA aptamer against IL-32 might effectively prevent the progression of inflammation caused by IL-32 associated TNF $\alpha$ expression.

\section{Experimental Section}

Preparation of IL-32 protein and random RNA library. Re- 
(A)

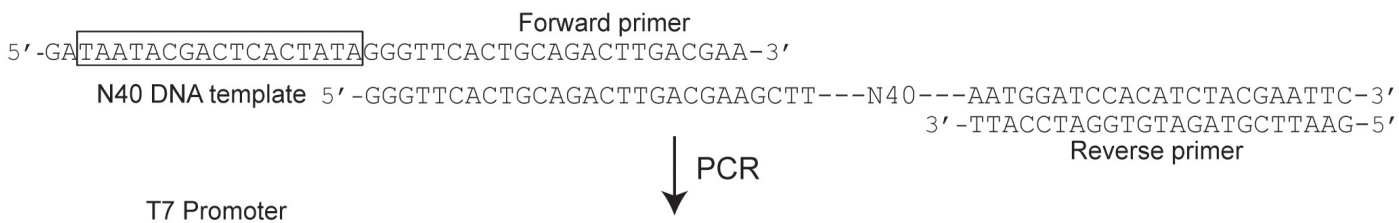

5' -GA PAATACGACTCACTATAGGGTTCACTGCAGACTTGACGAAGCTT---N40---AATGGATCCACATCTACGAATTC-3' 3' -CTATTATGCTGAGTGATATCCCAAGTGACGTCTGAACTGCTTCGAA---N40---TTACCTAGGTGTAGATGCTTAAG-5'

(B)

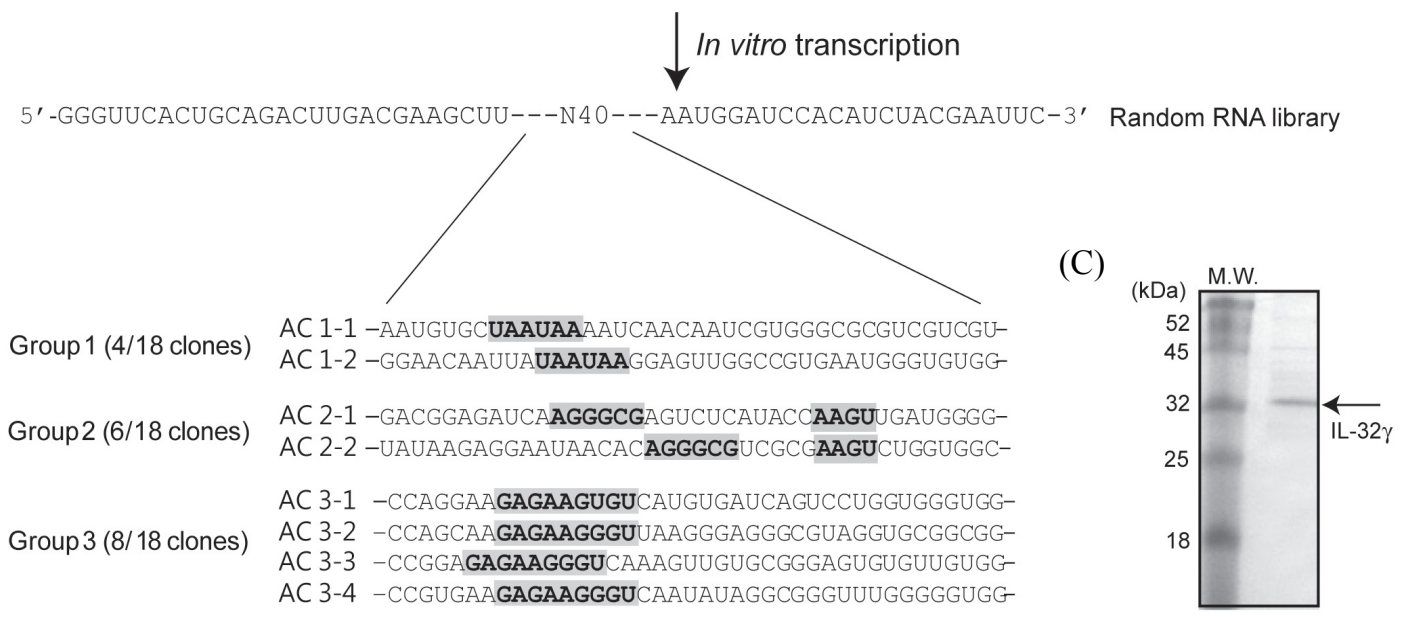

Figure 1. Preparation of oligonucleotides for SELEX and sequences of selected RNA. (A) The sequence of the RNA pool for in vitro selection is shown. A random RNA library was obtained by in vitro transcription of the DNA template, which contains 40 random nts. (B) The RNAs selected after the tenth round of SELEX were sequenced and grouped based on sequence similarity. Each group had conserved sequences as indicated by shaded nucleotides. (C) The purified recombinant IL-32 $\gamma$ protein was analyzed by $12 \%$ SDS-PAGE analysis with Coomassie Brilliant Blue staining and was found to be a single band with a molecular weight of $35 \mathrm{kDa}$ (arrow).

combinant human IL-32 $\gamma$ protein harboring $6 x$ His-tag at the $\mathrm{N}$-terminus was expressed in Escherichia coli and purified by TALON affinity chromatography (Invitrogen, San Francisco, CA) and subsequent size-exclusion chromatography (Superdex G75, GE Healthcare, Piscataway, NJ), as described previously. ${ }^{22}$ The purified recombinant IL-32 $\gamma$ protein that was found to be about $35 \mathrm{kDa}$ upon SDS-PAGE analysis with Coomassie Brilliant Blue staining (Fig. 1C) was subjected to RNA aptamer screening. The random RNA library used for selection of IL-32 specific RNA aptamers (Fig. 1A) was prepared as described previously. $^{23}$

In vitro selection of IL-32 specific RNA aptamers. In vitro selection was conducted using the purified His-tagged IL-32 $\gamma$ protein and the generated random RNA library. The random RNA library $(6 \mu \mathrm{g})$ was preincubated with $100 \mu \mathrm{L}$ of Ni-NTA Sepharose beads (GE Healthcare) in $100 \mu \mathrm{L}$ of binding buffer (20 mM sodium phosphate, $500 \mathrm{mM} \mathrm{NaCl}$ and $20 \mathrm{mM}$ imidazole, $\mathrm{pH}$ 7.4) for $15 \mathrm{~min}$ at room temperature with occasional shaking. The RNA-bead complexes were briefly centrifuged and discarded to remove RNAs with nonspecific binding to Sepharose bead. The SELEX procedure was conducted using Ni-Sepharose His SpinTrap columns (GE Healthcare). The resin was washed and equilibrated with binding buffer, after which His-tagged IL-32 $\gamma$ protein $(50 \mu \mathrm{g})$ that was pre-incubated with the binding buffer for $15 \mathrm{~min}$ at room temperature was loaded onto the column and further eluted with the binding buffer. After the column was washed three times with binding buffer, $100 \mu \mathrm{L}$ of the pre-cleared random RNA library was load- ed onto the column. The column was washed three times with the binding buffer to remove RNA molecules that did not bind to the protein. Next, the bound RNA was eluted using elution buffer ( $20 \mathrm{mM}$ sodium phosphate, $500 \mathrm{mM} \mathrm{NaCl}$ and $300 \mathrm{mM}$ imidazole, $\mathrm{pH}$ 7.4), collected and then purified by phenol-chloroform extraction and ethanol precipitation. The recovered RNAs were reverse transcribed with RT pre-mix ${ }^{\mathrm{TM}}$ (Intron Biotechnology, Gyeonggi-do, Korea) and amplified by PCR with Taq DNA polymerase (SunGenetics, Daejeon, Korea), and the amplified DNA was subjected to the in vitro transcription to generate an RNA library for the next round. Following the $10^{\text {th }}$ round, the amplified cDNA was cloned into a linearized pGEM T-Easy vector (Promega, Madison, WI). Subcloning and transformation was carried out using $E$. coli, after which plasmid DNA that was isolated from each clone was sequenced. Each RNA aptamer was prepared by in vitro transcription using the amplified cDNA or respective subcloned plasmid as a template.

Measurement of RNA aptamer binding to $\mathbf{I L - 3 2}$ protein. To comparatively analyze the binding affinity of each RNA aptamer, semi-quantitative RT-PCR was carried out to analyze RNAs eluted from the column after binding the individual RNA aptamer candidate to IL-32 protein. Specifically, $70 \mathrm{ng}$ of IL-32 protein (2.0 pmole) was loaded and immobilized onto a Ni-Sepharose column, and the exact amount (20 pmole) of RNA aptamer candidate was poured into the column, as performed in the SELEX process. The column containing the RNA-protein complex was then washed three times with $200 \mu \mathrm{L}$ of the binding buffer, after which the eluents were collected as flow-thru. 
The IL-32 bound RNAs were then eluted from the column by three consecutive washes with $200 \mu \mathrm{L}$ of the elution buffer. RNAs from the flow-thru and the IL-32 bound RNAs were then extracted by phenol-chloroform and ethanol precipitation, and the extracted RNAs from each elution were subjected to RTPCR using the aptamer specific primers described above. The conditions for the PCR were as follows: 18 cycles of $95^{\circ} \mathrm{C}$ for $20 \mathrm{~s}, 55^{\circ} \mathrm{C}$ for $20 \mathrm{~s}$, and $72{ }^{\circ} \mathrm{C}$ for $30 \mathrm{~s}$. Agarose gel electrophoresis was carried out to confirm that DNA was amplified from each eluant and the amount of amplified DNA was quantified using the Gel-Pro analyzer software (Media Cybernetics, Bethesda, MD).

Alternatively, the electrophoretic mobility shift assay (EMSA) was conducted to monitor the binding affinity of the selected RNA aptamer against IL-32. A fixed concentration of the selected RNA aptamer $(100 \mathrm{nM})$ was incubated with the target protein at various concentrations $(10 \sim 500 \mathrm{nM})$ in $20 \mu \mathrm{L}$ of binding buffer for $15 \mathrm{~min}$ at room temperature. The RNA-protein complex was subjected to $8 \%$ non-denaturing polyacrylamide gel electrophoresis and the resolved RNAs were then identified by ethidium bromide staining. Next, the protein-bound RNA was quantified and analyzed using the Gel-pro analyzer software, and a binding curve was generated which was fitted to a hyperbolic equation using Sigma plot (SPSS, Chicago, IL).

Cell culture and RNA aptamer transfection. Human lung epithelial cells with IL-18 receptor $\beta$ chain expression (A549$\mathrm{R} \beta$ ) that were cultured, as previously described, ${ }^{2}$ in DMEM (HyClone Laboratories, Logan, UT) supplemented with 1\% penicillin-streptomycin and 10\% fetal bovine serum. Cells were grown in a humidified atmosphere of $5 \% \mathrm{CO}_{2}$ at $37{ }^{\circ} \mathrm{C}$. Cells $\left(2 \times 10^{5}\right.$ cells/well $)$ were seeded in 6 -well plates in $1.0 \mathrm{~mL}$ of culture medium and then grown for additional $48 \mathrm{hrs}$. Transfection of IL-32 RNA aptamer or negative control RNA was carried out in 6-well plates with cells reaching $60 \sim 70 \%$ confluence. The plated cells were then washed twice with phosphate-buffered saline (PBS) and placed in $500 \mu \mathrm{L}$ of serum-free medium (DMEM) prior to transfection. RNA aptamers of given concentrations (10 to $100 \mathrm{nM}$ ) were mixed with Lipofectamine ${ }^{\mathrm{TM}} 2000$ (Invitrogen) and transfection was conducted as previously suggested by the manufacturer. For the case of IL-32 induction with IL-1 $\beta$ stimulation, cells were treated with $30 \mathrm{ng} / \mathrm{mL}$ of hIL-1 $\beta$ (R\&D systems, Minneapolis, MN) 24 hrs after transfection. The cells were then further incubated for $36 \mathrm{hrs}$ after IL-1 $\beta$ treatment or incubated for $60 \mathrm{hrs}$ after RNA transfection without IL-1 $\beta$, after which they were trypsinized and harvested by centrifugation. The cell pellets and supernatants were then collected for western blotting assay and ELISA, respectively.

Westem blotting assay. Harvested cell pellets were resuspended and lysed, and the total proteins were obtained and quantified using protein assay reagent (Bio-Rad, Hercules, CA). A

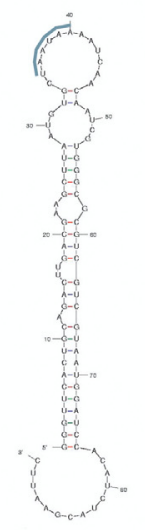

AC1-1

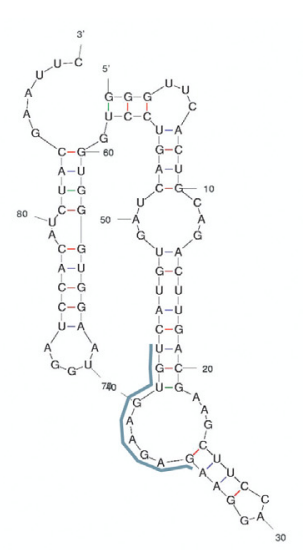

AC3-1

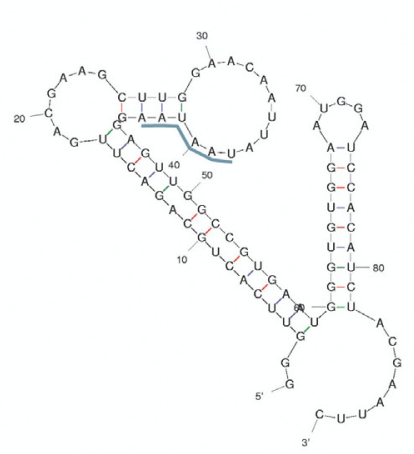

AC1-2

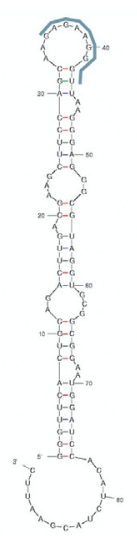

AC3-2

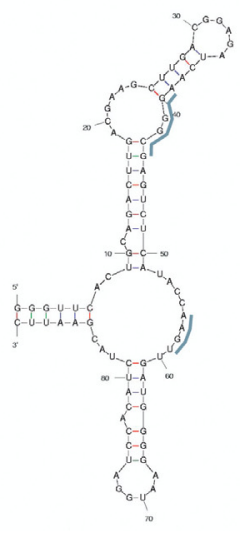

AC2-1

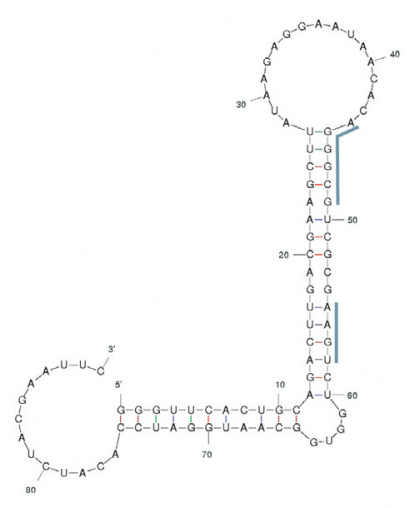

AC2-2

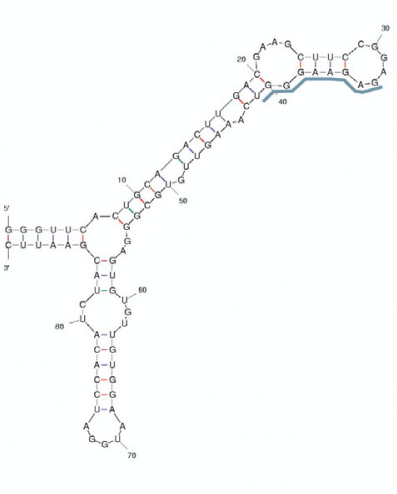

AC3-3

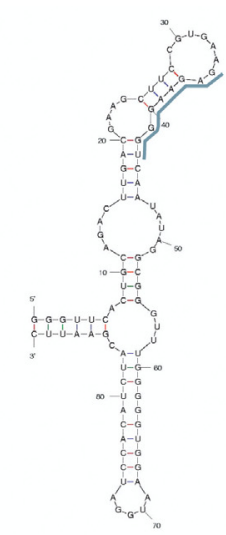

AC3-4

Figure 2. Predicted secondary structures of IL-32 RNA aptamer candidates. Predicted secondary structures of eight different sequences of IL-32 aptamer candidates (AC) obtained using Zuker's M-Fold program are shown. The conserved sequences in each group are indicated by thick lines. 
total of $25 \mu \mathrm{g}$ of proteins were loaded onto a $12 \%$ SDS-PAGE and then transferred to nitrocellulose membrane (Whatmann, Piscataway, NJ). The membrane was then blocked with 5\% nonfat milk and probed with the following primary antibodies: anti-IL-32 $\gamma$ (prepared as described previously ${ }^{12}$ ), anti-TNF $\alpha$ (Abcam, Cambridge, UK), and anti- $\beta$-actin (Santa Cruz Biotechnology, Santa Cruz, CA). After washing, the membrane was incubated with secondary antibodies conjugated with enzymeperoxidase (Gendepot, Barker, TX) at a 1:10,000 dilution for 1 $\mathrm{hr}$ at room temperature, then developed using the enhanced chemical luminescence system (Intron Biotechnology).

Enzyme-linked immunosorbent assay (ELISA). Collected cell culture supernatants that contain excreted human TNF $\alpha$ were assayed using a commercially available human cytokine ELISA kit (R\&D Systems, Minneapolis, MN) for the liquidphase enhanced chemiluminescence (ECL) method according to the vendor's instructions. The amount of chemiluminescence from $300 \mu \mathrm{L}$ of the supernatant was measured at $450 \mathrm{~nm}$ using an ELISA reader (Photoread, Berthold, Germany). All values (expressed as $\mathrm{pg} / \mathrm{mL}$ ) represent the mean \pm standard deviation of triplicate independent experiments.

\section{Results and Discussion}

Selection of RNA aptamers specific to IL-32 protein. To generate RNA aptamers against IL-32, the SELEX method was adopted for in vitro selection of target specific ligands. Recombinant human IL-32 $\gamma$ protein was expressed and purified to homogeneity as determined by SDS-PAGE and western blot analysis (data not shown). The SELEX process was started from the pool of the RNA library $\left(\sim 10^{24}\right.$ nucleic acid sequences) that was generated via PCR and in vitro transcription of DNA templates harboring random sequences of $40 \mathrm{nts}$ (Fig. 1A). To select RNA aptamers with a high affinity to the target protein, 10 iterative rounds were conducted by increasing the stringencies of RNA-binding to the target protein as the rounds progressed. Starting from the 4th round, a more stringent condition was employed by reducing the protein concentration at each subsequent round: $25 \mu \mathrm{g}$ (rounds 4-5), $10 \mu \mathrm{g}$ (rounds 6-7), $5.0 \mu \mathrm{g}$ (rounds $8-9$ ), and $2.5 \mu \mathrm{g}$ (round 10).

After ten cycles of selection, the round ten pool was cloned and 18 individual aptamer clones were sequenced. Sequence alignment of these clones enabled them to be categorized into three groups (Fig. 1B). Each group had a characteristic sequence of clustered nucleotides in the middle of the random sequences region, in which there was an AG-rich conserved sequence of nine nucleotides [GAGAAG(G/U)GU] and ten nucleotides [AGGGCG] followed by [AAGU] in group 3 and 2, respectively. Besides these groups, four clones among 18 subclones were found to contain AU-rich sequences of six nucleotides [UAAUAA] (Group 1). The secondary structures of eight different RNA sequences identified in the tenth RNA pool were predicted using the M-Fold program based on the Zuker algorithm. ${ }^{24}$ Every RNA aptamer was predicted to contain several stem-loop structures, in which the conserved sequences largely reside at the loop or short stem flanking the loop regions in the secondary structures (Fig. 2). These results imply that the conserved sequences in each RNA aptamer candidate might be
(A)
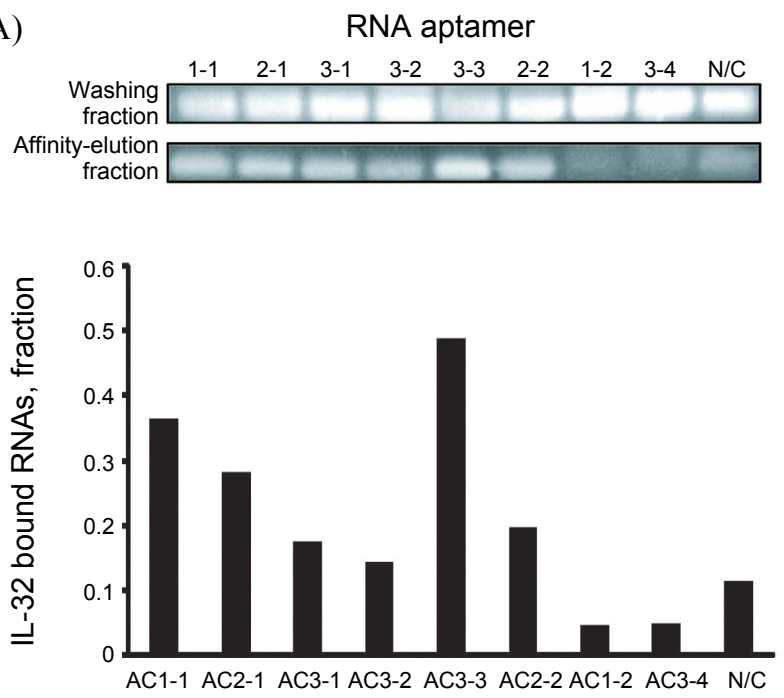

(B)
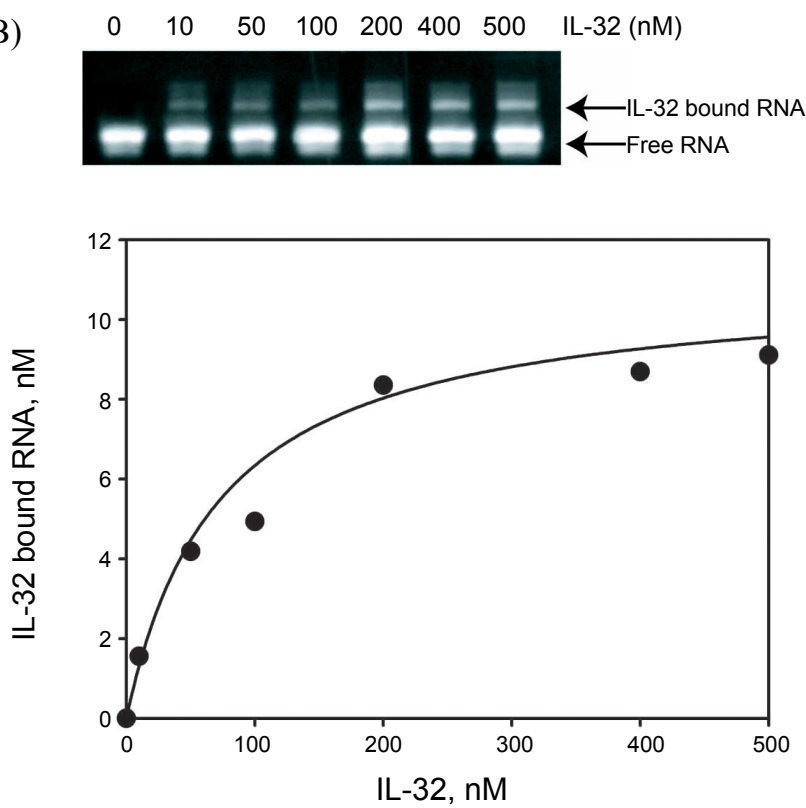

Figure 3. Measurement and confirmation of the binding affinity of aptamers. (A) Affinity measurement using RT-PCR was performed with a His spin trap column. A precise amount ( 20 pmole) of eight different RNA aptamer candidates and non-specific RNA (negative control, N/ C) was reacted with IL-32 protein (2.0 pmole) in the affinity column chromatography. RNA from the washing fraction and the affinityelution fraction was reverse transcribed and its cDNA was amplified. Ethidium bromide staining identified free RNA and protein-bound RNA in 2\% agarose gel electrophoresis. (B) RNA aptamer AC3-3 at $100 \mathrm{nM}$ was incubated with increasing concentrations of IL-32. The RNA-protein complex was subjected to $8 \%$ non-denaturing polyacrylamide gel electrophoresis and the resolved RNA was identified by ethidium bromide staining. The upper arrow indicates the RNAs bound to IL-32 protein and the bottom arrow indicates the free RNA. The amount of protein-bound RNA was calculated and graphed as a function of protein concentration, which was fit to the hyperbolic equation to provide $\mathrm{K}_{\mathrm{d}}$ of $78 \mathrm{nM}$.

exposed for interaction with the IL-32 protein. It has been shown that RNA aptamers for target proteins often contain a conserved sequence of several nucleotides in the loop structure. ${ }^{23,25}$ Thus, 
the conserved sequences residing at the RNA aptamer loop and stem could constitute a binding motif structure to IL-32, whereas the stem structure of any other sequence could have a stabilizing function on the RNA aptamer structure.

Binding affinity of RNA aptamers for IL-32 protein. To analyze the binding affinity of the eight aptamer candidates targeting the IL-32 protein, we carried out semi-quantitative RT-PCR for RNAs bound to the protein using a strict amount of RNA aptamer candidates and IL-32 protein (Fig. 3A). For this method, we used the His spin trap column in conjunction with the SELEX process for immobilization of IL-32 protein, and PCR was limited to 18 cycles to avoid saturation of cDNA amplification. The flow-through and affinity-eluted RNA fractions were then collected and subjected to RT-PCR. The loaded RNAs were then partitioned into the washing fraction that was unbound to the protein and the affinity-elution fraction that bound to the protein. As expected, the amount of RNAs bound to IL-32 protein was negligible when compared to the negative control RNA containing specific sequences to irrelevant viral protein. ${ }^{23}$ In contrast, some RNAs obtained at the tenth round of SELEX exhibited significant binding activity by showing an enriched amount of RNA in the affinity-elution fraction. Among these RNA aptamer candidates, aptamer clone AC3-3 was the most prominent one able to bind to the target protein and was therefore selected as the highest affinity aptamer for further study.

A gel retardation assay was conducted to measure the binding affinity of RNA aptamer AC3-3 for the IL-32 protein (Fig. 3B). Increasing amounts of IL-32 protein were mixed with a fixed amount of RNA aptamer AC3-3 (100 nM). The RNA aptamer was found to bind to the IL-32 protein in a dose-dependent manner, as indicated by the increased intensity in the proteinbound RNA band. After quantification of the RNA bands, a hyperbolic equation was applied to fit the binding of RNA to the protein. The fit provided an apparent dissociation constant $\left(\mathrm{K}_{\mathrm{d}}\right)$ of $78 \mathrm{nM}$ for the RNA aptamer AC3-3 with a saturation in binding above $200 \mathrm{nM}$ of IL-32 protein. About $10 \%$ of the RNA aptamer was bound to the protein without a further increase in binding at a higher concentration of protein, suggesting that only a fraction of the RNA aptamer might be structurally competent for binding to IL-32 protein under the conditions used in the assay. We are currently investigating a method to increase the population of RNA aptamers that is competent for binding to the target protein by thermally resolving and reconstituting the RNA structures.

Antagonistic activity of RNA aptamer against IL-32. IL-32 is well known as an inducer of proinflammatory cytokines such as TNF $\alpha$ and IL-1 $\beta$. To determine if the high affinity RNA aptamer AC3-3 generated in this study was capable of antagonizing the function of IL-32 protein in cells that endogenously produce IL-32, we conducted Western blot assay to monitor the expression of proinflammatory cytokines after transfection of the selected RNA aptamer in cells. The lung cancer cell line A549 variant (A549-R $\beta$ ) that is the human lung carcinoma A549 cell line with an IL-18 receptor $\beta$ chain (IL-18R $\beta$ ) expression was used to test the efficacies of AC3-3 RNA aptamers in cells. The stable clones of the IL-18R $\beta$ gene transfected A549 cells (A549-R $\beta$ ) that are responsive to IL-18 or IL-1 $\beta$ exhibit upregulation of several cytokines, including IL-6, IL-8, and IL-32.
(A)

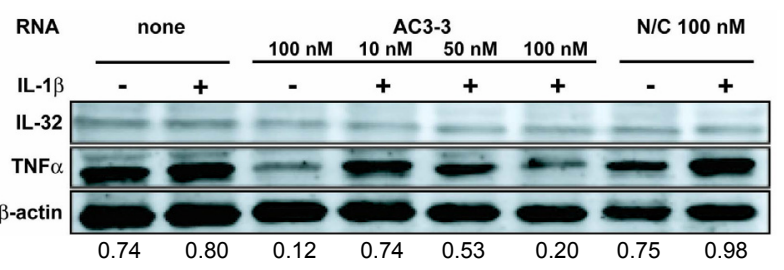

(B)

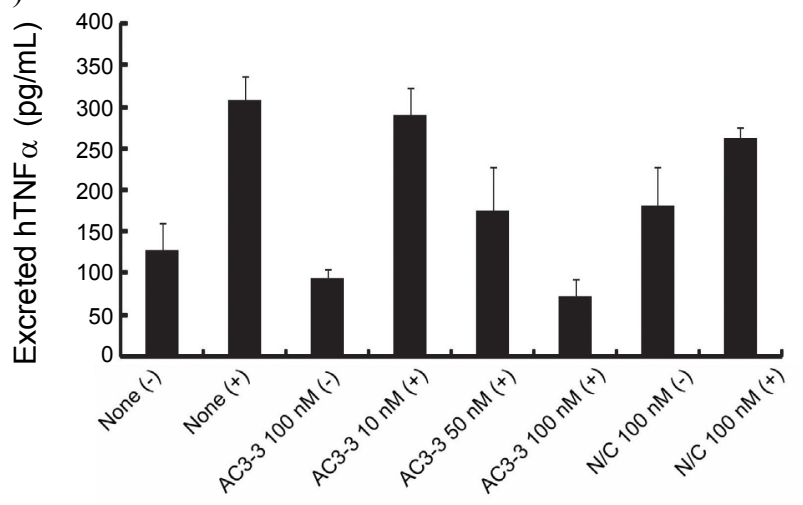

Figure 4. Antagonistic activity of RNA aptamer AC3-3 against IL-32. Lung cancer cells that expressed IL-18 receptor (A549R $\beta$ ) were transfected with the RNA aptamer AC3-3 at increasing concentrations or the negative control RNA of irrelevant sequences (N/C) at $100 \mathrm{nM} .24$ hrs after RNA transfection, IL-1 $\beta(30 \mathrm{ng} / \mathrm{mL})$ was added to the cells to induce proinflammatory cytokines IL-32. The IL-32-induced TNF $\alpha$ production was examined after $36 \mathrm{hr}$ incubation. (A) The cell extracts were prepared and analyzed by Western blotting analysis with anti-IL32 and anti-TNF $\alpha$ antibodies. To confirm equal loading, the blots were re-probed with anti- $\beta$-actin antibody. The amounts of TNF $\alpha$ protein were determined by densitometry and were normalized to the $\beta$-actin protein, as shown by the numbers below the gel image. (B) IL-32-induced TNF $\alpha$ secretion in the culture media was measured by ELISA. Cell culture supernatant derived from the cell harvest in (A) was analyzed by liquid-phase ECL (enhanced chemiluminescence) system for human TNF $\alpha$ excreted in media. The signs in the parenthesis represent the presence or absence of IL-1 $\beta$ in the cell growth media. All experiments were carried out in triplicate and the error bars represent the S.D.

First, RNA aptamer AC3-3 at three different concentrations was transfected into the A549-R $\beta$ cells stimulated with IL-1 $\beta$ and the expression level of cytoplasmic IL-32 and TNF $\alpha$ was monitored (Fig. 4A). TNF $\alpha$ production in cells with or without IL-1 $\beta$ stimulation was substantially reduced by $100 \mathrm{nM}$ of the RNA AC3-3 transfection, whereas the level of IL-32 protein in the cells was not affected under this condition. Suppression of TNF $\alpha$ expression by the AC3-3 aptamer was not pronounced at an AC3-3 dosage lower than $50 \mathrm{nM}$. In contrast, the irrelevant RNA with nonspecific sequences did not reduce TNF $\alpha$ expression in cells, regardless of IL- $1 \beta$ stimulation. These results indicate that the RNA aptamer specific to IL-32 functionally antagonizes IL-32 and subsequently reduces $\mathrm{TNF} \alpha$ expression without affecting IL-32 expression level in the cells, demonstrating the inhibitory efficacy of the RNA aptamer against IL-32.

Next, we examined the inhibitory effect of AC3-3 against IL-32-induced TNF $\alpha$ release from A549-R $\beta$ cells. Cell culture supernatants obtained from cells harvested for western blot 
assay were assessed for human TNF $\alpha$ production using ELISA (Fig. 4B). IL-32-induced TNF $\alpha$ secretion was reduced with the AC3-3 aptamer transfection in a dose-dependent manner. At high concentrations of AC3-3 aptamer $(100 \mathrm{nM})$, TNF $\alpha$ production was reduced by nearly $75 \%$ when compared with the untreated control. In contrast, the IL-32-induced TNF $\alpha$ release was not diminished by the irrelevant RNA, regardless of IL- $1 \beta$ stimulation. These data suggest that inhibition of the IL-32induced TNF $\alpha$ secretion was caused by specific activity of the IL-32 antagonizing RNA aptamers in cells.

Taken together, we demonstrated that the RNA aptamer AC33 specifically antagonizes the function of IL-32 to induce its cognate proinflammatory cytokine, TNF $\alpha$. The exact role of IL-32 in proinflammatory disorders has not been fully elucidated due to the lack of selective antagonists against IL-32. Thus, the RNA aptamer AC3-3 generated in this study can be used as a potent and selective antagonist against human IL-32 to probe potential roles of IL-32 in immune function as well as to treat chronic inflammatory diseases if appropriate RNA delivery agents are developed.

Acknowledgments. We thank Dr. Soohyun Kim (Konkuk University, Seoul, Korea) for a generous gift of A549-R $\beta$ cells. This work was supported by an NRF grant funded by the MEST (2009-0071058, 2010-0019306), a grant from the Industrial Technology Development, Ministry of Knowledge Economy (10032113), and a grant from the National R\&D Program for Cancer Control, Ministry for Health, Welfare and Family affairs (0920080), Republic of Korea. D-Y.Y is supported in part by the Priority Research Centers Program (2009-0093824).

\section{References}

1. Dahl, C. A.; Schall, R. P.; He, H. L.; Cairns, J. S. J. Immunol. 1992 , 148, 597.

2. Kim, S. H.; Han, S. Y.; Azam, T.; Yoon, D. Y.; Dinarello, C. A.
Immunity 2005, 22, 131.

3. Kobayashi, H.; Lin, P. C. Cytokine 2009, 46, 351.

4. Dinarello, C. A.; Kim, S. H. Ann Rheum Dis 2006, 65 Suppl 3, iii61.

5. Bombardieri, M.; McInnes, I. B.; Pitzalis, C. Expert Opin Biol Ther 2007, 7, 31.

6. Netea, M. G.; Azam, T.; Ferwerda, G.; Girardin, S. E.; Walsh, M. et al. Proc. Natl. Acad. Sci. USA 2005, 102, 16309.

7. Netea, M. G.; Lewis, E. C.; Azam, T.; Joosten, L. A.; Jaekal, J. et al. Proc. Natl. Acad. Sci. USA 2008, 105, 3515.

8. Goda, C.; Kanaji, T.; Kanaji, S.; Tanaka, G.; Arima, K. et al. Int. Immunol. 2006, 18, 233.

9. Novick, D.; Rubinstein, M.; Azam, T.; Rabinkov, A.; Dinarello, C. A. et al. Proc. Natl. Acad. Sci. USA 2006, 103, 3316.

10. Shoda, H.; Fujio, K.; Yamaguchi, Y.; Okamoto, A.; Sawada, T. et al. Arthritis. Res. Ther. 2006, 8, R166.

11. Joosten, L. A.; Netea, M. G.; Kim, S. H.; Yoon, D. Y.; OppersWalgreen, B. et al. Proc. Natl. Acad. Sci. USA 2006, 103, 3298.

12. Kim, K. H.; Shim, J. H.; Seo, E. H.; Cho, M. C.; Kang, J. W. et al. J. Immunol. Methods 2008, 333, 38.

13. Vakkila, J.; Lotze, M. T. Nat. Rev. Immunol. 2004, 4, 641.

14. Nishida, A.; Andoh, A.; Inatomi, O.; Fujiyama, Y. J. Biol. Chem. 2009, 284, 17868

15. Hong, J.; Bae, S.; Kang, Y.; Yoon, D.; Bai, X. et al. Cytokine 2010, 49, 171.

16. Scott, D. L.; Kingsley, G. H. N. Engl. J. Med. 2006, 355, 704.

17. Dinarello, C. A. J. Exp. Med 2005, 201, 1355.

18. Tuerk, C.; Gold, L. Science 1990, 249, 505.

19. Ellington, A. D.; Szostak, J. W. Nature 1990, 346, 818

20. Jayasena, S. D. Clin. Chem. 1999, 45, 1628.

21. Rhodes, A.; Deakin, A.; Spaull, J.; Coomber, B.; Aitken, A. et al. J. Biol. Chem. 2000, 275, 28555.

22. Seo, E. H.; Kang, J.; Kim, K. H.; Cho, M. C.; Lee, S. et al. J. Microbiol. Biotechnol. 2008, 18, 1606.

23. Jang, K. J.; Lee, N. R.; Yeo, W. S.; Jeong, Y. J.; Kim, D. E. Biochem. Biophys. Res. Commun. 2008, 366, 738.

24. Zuker, M. Methods Enzymol 1989, 180, 262.

25. Fukuda, K.; Vishnuvardhan, D.; Sekiya, S.; Hwang, J.; Kakiuchi, N. et al. Eur. J. Biochem. 2000, 267, 3685.

26. Li, W.; Yang, F.; Liu, Y.; Gong, R.; Liu, L. et al. Eur. J. Immunol. 2009, 39, 1019. 